\title{
THE ELECTRO-ENCEPHALOGRAPHIC LOCALIZATION OF INTRA-CRANIAL NEOPLASMS
}

\author{
BY \\ W. A. COBB \\ (From Hurstwood Park Neurosurgical Centre)
}

(RECEIVED 24TH August, 1944)

IT was first pointed out by Berger in 1929 that the presence of an intra-cranial tumour might cause changes in the electroencephalogram (e.e.g.), and since that time a considerable number of papers have been published which are concerned with various tumour effects and with methods for their localization. No recent paper has, however, discussed a fairly large series of cases, and this is my reason for adding one more to the number.

Technique.-The apparatus used was a 2-channel Grass ink-writing oscillograph, kindly loaned to this hospital by Professor E. D. Adrian. The use of only two channels for purposes of localization involves considerable difficulties, as well as expenditure of time, which are not present with three or more channels. Firstly, there is the simple point that more recordings are required to obtain equivalent coverage of the scalp; secondly, any given wave can be seen only in two positions, but ideally one should be able to study the distribution of any given potential change in as many positions as possible; thirdly, if amplitudes in three channels show a rising gradient, then it is probable that the origin of the disturbance is in the direction of the greatest amplitude, or, if the amplitude of the middle channel is lower than that of the outer ones, the disturbance probably arises between the middle pair of electrodes. Inferences of this sort are clearly not possible with only two channels, but the difficulty can be surmounted to some extent: 4 electrodes are placed in a row and recording is first done from $1-2,2-3$, and then from $2-3,3-4$; so long as $2-3$ remains fairly constant in both recordings they can be regarded as approximately equivalent to a simultaneous three-channel recording. This point has been stressed because there appears to be a tendency in America for manufacturers to offer single channel oscillographs to the profession, and timely warning may prevent such misguided efforts here.

The routine recording was from four antero-posterior lines of four electrodes, low along either side and about 1 in. lateral to the mid-line: this was followed in some cases by similar transverse recordings, and then by odd placements according to the findings. Recording was bipolar throughout, the method being essentially that devised by Walter. The electrodes were saline pads on silver chloride-silver, in Perspex mounts. The patients were for preference seated, but when their condition did not allow of this, were lying on an earthed metal trolley.

The material for these investigations consisted of 155 consecutive suspected intracranial spaceoccupying lesions. Among the earlier cases there was some degree of selection by the clinicians, the e.e.g's. being requested more particularly in cases of doubt, so that, for example, very few pituitary tumours are included in the earlier part of the series; the later cases are consecutive and unselected. From the 155 cases, 30 have been rejected because either the exact nature or localization of the lesion remained in doubt, or the patient's unco-operative state prevented an adequate recording. The remaining 125 lesions were fully confirmed in site and pathology, though not in all cases in extent.

\section{TABLE I}

\begin{tabular}{|c|c|c|c|c|c|c|c|c|c|}
\hline$A C E$ & $0-5$ & $6-10$ & $11-16$ & $16-20$ & $21-30$ & $31-40$ & $41-50$ & $51-60$ & over 60 \\
\hline No. & - & 2 & 3 & 10 & 20 & 32 & 30 & 19 & 9 \\
\hline
\end{tabular}

Distribution in Age Groups.

The sexes were fairly equally divided, with 69 males and 56 females. The distribution of age groups is shown in Table I.

\section{Analysis of results}

In Table II the lesions have been grouped partly on an anatomical and partly on a pathological basis. Of the 21 subtentorial tumours, only three were correctly lateralized. Although some claims have been made to localize cerebellar tumours by means of the e.e.g. (Smith, J. R., et al., 1940), its usefulness in this sphere appears more than doubtful. On the other hand, the record commonly shows a generalized abnormality which may be of confirmatory evidence in conjunction with the clinical data.

TABLE II

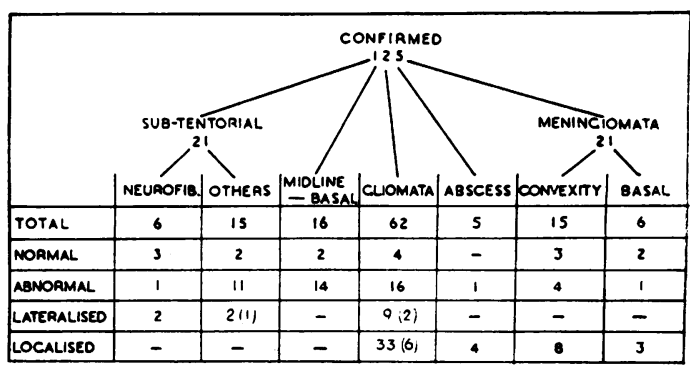

Analysis of Material. Normal-not definitely abnormal. Abnormal-but not lateralized. Lateralized -but not localized. Figures in parentheses-incorrectly lateralized or localized. 
he second group, of midline and basal tumours, is a mixture of pituitary, 3rd ventricle, brain-stem, and similarly situated lesions, some benign and some malignant. Localization in the usual sense is clearly not possible, so no attempt has been made to tabulate results, but this does not mean that suggestive and useful evidence has not been obtained.

The remaining lesions are all cerebral, in most cases approaching or reaching the free convexity; with the gliomata it is, of course, difficult to be sure of the exact relationships and extent of the tumour. Because of their defined boundaries, and also clinical importance, the meningiomata have been grouped separately, with the exception of two posterior fossa tumours which are included in the subtentorial group. Abscesses affect the e.e.g. in a similar manner to tumours, but on account of their greater acuity tend to show more and slower electrical activity.

\section{TABLE III}

\begin{tabular}{|c|c|c|c|c|c|}
\hline & NORMAL & ABNORM. & LATERAL. & LOCAL. & INCORRECT. \\
\hline NO. & 9 & 22 & 7 & 42 & 8 \\
\hline$\%$ & 10.2 & 25 & 8 & 47.8 & 9 \\
\hline
\end{tabular}

Analysis of corrected results in 88 cases. 62 gliomata, 5 abscesses and 21 meningiomata.

Table III shows the results obtained from the 88 "cerebral " lesions which might have been expected to be susceptible to localization. It will be seen that approximately only half were correctly localized, a figure which is considerably lower than that of some previous writers. This is, no doubt, in part due to inexperience-the percentage is tending to rise-and in part to the disabilities imposed by the use of only two channels.

Alpha Rhythm.-No exact analysis of the distribution of alpha frequencies has been attempted, but in Fig. 1 the percentage of cases falling into con-

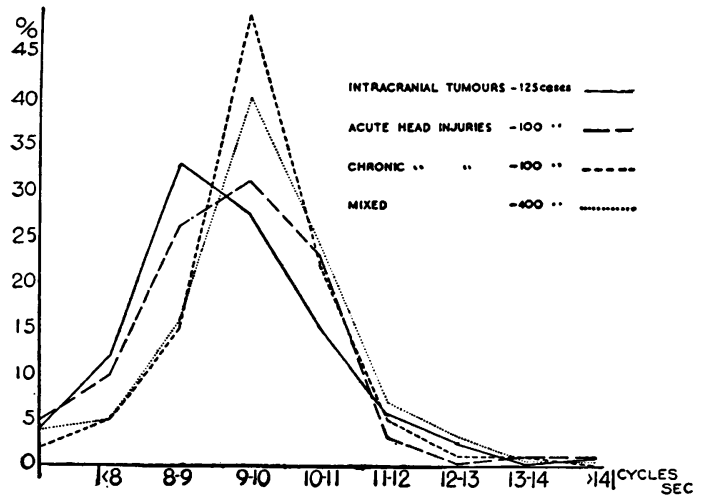

Fig. 1.-Distribution of Alpha frequencies shown as percentages of total in each group. There is a slightly greater tendency to slowing in the Tumour group than in either of the others.

secutive frequencies from $<8 / \mathrm{sec}$. to $>14 / \mathrm{sec}$. has been compared with similar findings in 100 acute (less than 3 weeks) head injuries, 100 chronic (more than 3 weeks) head injuries and 400 mixed cases of all types, including epileptics, psychoneurotics, etc., but excluding tumours and head injuries. It will be seen that in the tumour cases there is a greater tendency to slowing of the alpha rhythm than in either of the other groups.

Noticeable asymmetry. of the alpha rhythm is not a very marked feature of the records, though at times it is a useful aid to lateralization. Assessment is sometimes complicated by unilateral overshadowing of the alpha rhythm by gross slow activity. Ten patients showed an alpha rhythm of lower voltage or lesser regularity on the side of the lesion, while in two patients the reverse was the case. One of the latter had an acoustic nerve tumour, and the remainder had cerebral hemisphere lesions.

Raised Intracranial Pressure.-As in the clinical picture of an intracranial space-occupying lesion, so the e.e.g. may be composed of at least two fractions: that due to high intracranial pressure and that due to local changes. Although in the majority of cases it is not possible to separate these two effects with any certainty, it is nevertheless tempting to surmise that certain generalized effects are due to raised pressure. High pressure, in this sense, is really a misnomer, because the effects are found to have little direct relation to observed pressures, and for the more cogent reason that it has been demonstrated (Williams, 1939) that the withdrawal of c.s.f. from the ventricles has little effect on the record, while dehydrating the brain by means of hypertonic solutions does bring about an approach to normality. In other words, the " high pressure" record is really one of cerebral œdema.

From the e.e.g. standpoint the "high pressure" record is probably seen in its purest form in posterior fossa lesions, particularly small mid-line tumours blocking the 4th ventricle or aqueduct: such tumours being unlikely to give rise to any local effect. Between this "pure" effect and that so frequently seen in conjunction with localizable cortical lesions, it is not possible to draw any line. Despite statements to the contrary I do not believe there is any characteristic feature of the "high pressure" record by which it can be recognized with certainty. The abnormal waves are generalized, usually uniformly so, they are slow, though not necessarily very slow; fast waves have not been noted to be of special significance. A range, in a single record, from $<1 / \mathrm{sec}$. to $7 / \mathrm{sec}$. has been seen, but it is more commonly around $2-4 / \mathrm{sec}$; occasionally the waves have been rhythmic and more or less sinusoidal, but this is very exceptional and the usual impression is one of great irregularity. Amplitude is seldom very high and often less than that of the alpha rhythm.

As mentioned above, there is no direct correlation between the height of the c.s.f. pressure and the degree of abnormality of the record. One patient with a 6 weeks' history of blurred vision, and an initial c.s.f. pressure of over $300 \mathrm{~mm}$. had a normal record. Nor does the presence or degree of papillœdema appear to bear much relationship 
to the degree of abnormality of the record. There is greater correlation with the patient's state of consciousness, though even here the parallelism is by no means exact.

In general, in the absence of toxæmia or recent head injury, a record showing diffuse random slow activity of moderately high voltage is likely to be due either to " high pressure" or to cortical atrophy or vascular disease. The distinction is often possible on clinical grounds, but in cases of doubt it might be advantageous to apply Williams' technique of dehydrating the brain. I have not used the method for this purpose, but have found it satisfactory for separating high pressure from tumour effects in certain cases.

Local Effects.-These cannot be distinguished with certainty from the generalized effects of high pressure except by their focal or unilateral distribution, but high amplitudes and very slow frequencies are uncommon as pressure effects. The usual tumour record is a mixture of slow frequencies, sometimes quite haphazard, more often with one or more fairly regular frequency predominating, and sometimes occurring in well-defined bursts of almost sinusoidal form. Although perhaps it is true to say that the typical tumour record is one of very slow irregular waves of fairly high amplitude, the minimal appearance of only moderately slowed waves is not uncommon, and there is no feature by which their neoplastic causation can be forecast with certainty. Recent focal head injury, localized cortical atrophy, cerebral hæmorrhage or thrombosis and similar disruptive lesions may all produce the same type of record and distinction may not be possible on the evidence of a single recording: repetition at intervals, showing progression or regression of the electrical changes, will usually make the diagnosis clear.

In the common type of record in which there is a mixture of several frequencies, as opposed to a succession of completely irregular waves, it is sometimes possible to locate the separate origins of some of the frequencies; it seems at least probable that each frequency has a separate origin, rather than that the pace set by a common pacemaker is varied.

Localization.-The method of localization to be desired is the finding of phase-reversal of the slow waves, but this is by no means always achieved: 11 of the 62 gliomata were localized in this way, 2 of the 5 abscesses, and 7 of the 22 meningiomata. Although phase reversal gives the most definite indication of the source of the slow waves, this does not necessarily coincide with the site of the tumour, though it is usually in close anatomical association with it.

Walter (1937) has pointed out that in such cases. an area of cortical œdema may coincide with the delta focus - in one such case phase-reversal occurred some $5 \mathrm{~cm}$. posterior to the position of the tumourand the clinical signs may even be associated with the œdematous area rather than with the tumour site.

Another not uncommon finding is that of a delta focus associated with a deep central lesion. The delta waves must be cortical in origin, the cortex either being deprived of control by deeper structures. or driven by them at an abnormal rhythm. In one case a small glioblastoma of the posterior end of the corpus callosum gave rise to fairly regular $3 / \mathrm{sec}$. waves with phase-reversal in the region of the inion, while another is recorded below:

Case 1.-D. S., a girl of 15 who had a six weeks' history of headaches, associated for the last two weeks with vomiting. At the time of admission she had her first attack, lasting 2-3 hours, of diplopia on looking to the left. There was nothing of significance in her past or family histories.

When examined she had: some stiffness of the neck, bilateral papillœdema with 3-4D of swelling, slight right external rectus palsy, some left facial weakness, hypotonus of all limbs with inco-ordination of the right arm and leg. The tendon reflexes were all diminished but symmetrical, and the plantar responses were flexor.

The e.e.g. findings (Fig. 2) were: (1) An 8/sec. alpha rhythm, largely masked by other rhythms. (2) A $2 /$ sec. rhythm, tending to occur in fairly regular bursts of high voltage (150 micro volts) and showing phase reversal in both parasagittal parieto-occipital positions. It was temporarily inhibited by eye-opening. (3) A fairly continuous diffuse $6 / \mathrm{sec}$. rhythm of moderate voltage.

From these findings it was concluded that the lesion was probably a mid-line one involving the visual cortices, though indirect pressure from below the tentorium was not ruled out. Clinically the case was regarded as a posterior fossa lesion, and accordingly this area was explored, but no abnormality was found. Three weeks later a ventriculogram showed a large filling defect in the posterior part of the 3rd ventricle. A right ventriculostomy was done and the 3rd ventricle explored,

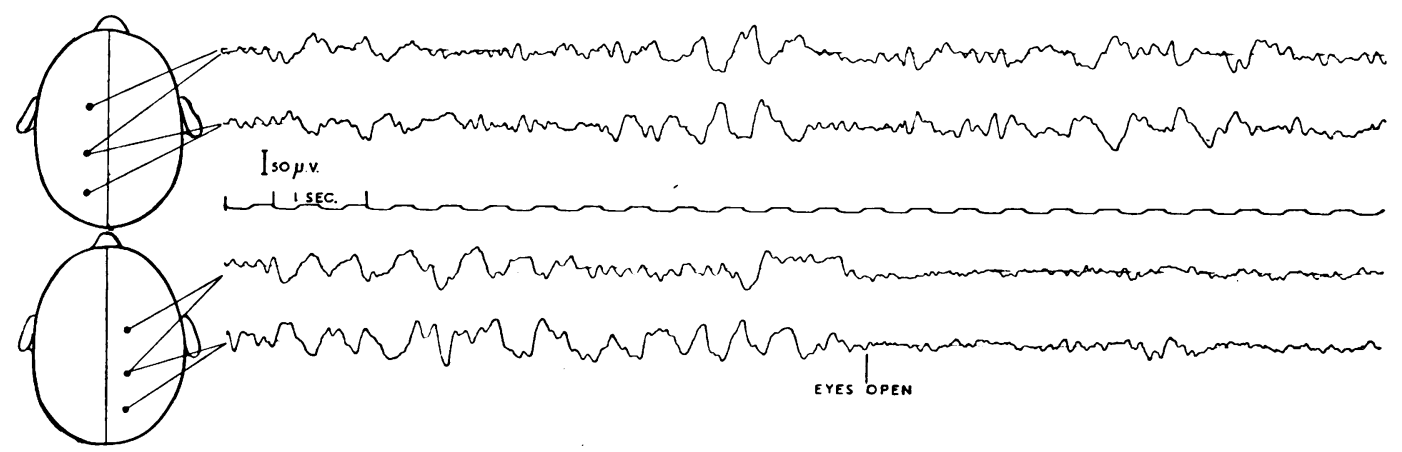

FIG. 2.-Case 1. Showing phase-reversal of $2 / \mathrm{sec}$. waves in both parietal areas. On the right side the effect of eye opening is shown. There is also a well-defined 6/sec. rhythm. 
revealing a fairly large spherical tumour arising from the region of the posterior commissure. A partial removal was attempted, but the patient died shortly after the operation. The tumour was an astrocytoma rising from the posterior commissure, with limited extension into surrounding structures.

In the type of record in which several frequencies are present it is usually the slowest which is of greatest significance, and phase-reversal of it correspondingly more so. Sometimes phase-reversal of two slow frequencies occurs in different positions, in which case that of the slower is usually of the greater significance, though one's conclusion may be modified by the presence of the faster; more will be said about this later, in connection with the 4-7/sec. frequency band.

There must, presumably, be a point or points, at which phase-reversal of any frequency occurs, but in many cases either the search for it is inadequate or it is inaccessible. In these circumstances reliance must be placed on finding the site of maximum intensity of the slow waves. It is difficult to define what is meant by "maximum intensity" as it involves a somewhat subjective assessment of amplitude, number, and possibly "slowness" of the abnormal waves: when it is considered that there may be a generalized background having very similar characteristics to those of the local disturbance it is apparent that this method may be by no means easy to apply; nevertheless very satisfactory localizations can be made by it, sometimes of surprisingly small extent.

Silent Areas.-Early in the history of the e.e.g. it was established (Foerster and Altenburger, 1935; Walter, 1936, 1937) that tumour tissue is electrically inert, and as a corollary, silent areas over superficial tumours have been described. In the present series they have only been convincingly demonstrated on two occasions; allowance should be made for the obvious difficulty of such a demonstration on two channels. In one, a small cyst was covered by about 1 in. of macroscopically normal tissue; in the other a large para-torcular meningioma corresponded with the silent area. In the first case, if not in both, it seems probable that what was found was an area of equipotential rather than a truly silent one.

Parasagittal Tumours.-It will be seen that 9, or 10.25 per cent. of the "accessible" tumours gave records which were not definitely abnormal. Two of them were basal tumours, and the remaining seven were all parasagittal, in part at least (the gliomata were, of course, of ill-defined extent). The difficulty of localizing parasagittal tumours has been noted (Gibbs and Gibbs, 1941), but such striking confirmation was unexpected, and is difficult to explain. It has been suggested that this difficulty is consistent with the routine practice of anteroposterior recording, and might be lessened by the use of transverse leads. This may perhaps be so, but does not account for the absence of definite abnormality. While it is possible that certain parts of the brain are incapable of producing delta waves, perhaps through lack of a pace-making mechanism, it is hardly plausible to suppose the entire parasagittal cortex to be in such marked distinction from the adjacent cortex with which its several areas are cytologically and functionally allied. While rejecting these two possible explanations it must be confessed that no better alternative is offered.

Three of the above seven tumours were meningiomata; this rather high proportion is in keeping with the general observation that on the whole they cause less electrical disturbance than malignant tumours, or, in slightly different form: the degree of disturbance is proportional to the rate of growth. There are very marked exceptions to this rule, but it is a fairly satisfactory approximation.

Hyperventilation.-This was done in a number of the cases, but was impossible or inadvisable in the remainder. The usual procedure was to overbreathe for three minutes, recording for the last half-minute, and a period after the return to normal breathing depending upon the observed result. As a rule it was impracticable to pay much attention to the patient's blood-sugar level which, for routine purposes, implies the avoidance of recording too soon after a meal.

Hyperventilation was performed in 43 cases in all. Of these, 29 showed substantially no change, and 11 some increase either in irregularity or amplitude, or both, often with some general tendency to slowing. The remaining 3 gave more definite abnormal responses. In one a period of gross slow activity was terminated by a syncopal attack and was almost certainly circulatory in origin. The other two were both brain-stem gliomata; one showed a prolonged bilateral $2 \frac{1}{2}-3 / \mathrm{sec}$. response, and the other a unilateral $5 /$ sec. discharge; there was nothing in the clinical state of the latter to explain the unilaterality, and the resting record was normal.

In general it can be said that the commonest result of hyperventilation in tumour cases is a tendency towards accentuation of those abnormal features already present, and that little, if any, assistance is to be expected from it.

Theta Rhythm.-Discussion of the group of midline and basal tumours has been postponed to this point because a new conception of some importance is involved. Among the tumours of this group are eight of the pituitary or suprasellar region and one epidermoid of the middle fossa, eroding the body of the sphenoid; that is to say: nine tumours lying in and around the pituitary fossa and involving, to great or less extent, the nervous structures in immediate apposition to it. With one exception (a pituitary tumour present for at least 34 years) these cases all showed a more or less well-marked pre-central or central rhythm within the range of 4-7/sec. Grey Walter, in a verbal communication to the E.E.G. Society, April, 1943, suggested that there might be some special association between the 4-7/sec. frequency band and tumours of the basal ganglia; he has recently (E.E.G. Society Meeting, February, 1944) reaffirmed this belief on the basis of thirty cases, confirmed at operation or post 
mortem, and proposes that the letter $\ominus$ should signify this rhythm.

The present material amply supports these views. In the eight cases mentioned above the rhythm has always been bilateral, though not necessarily symmetrical, and in some was associated with other rhythms; in such cases the tumours were found to have their greatest projection towards the side on which the Theta rhythm was most marked.

Case 2.-P. H., male, age 38. This patient had a 10 years' history of headaches, at first severe and associated with vomiting, but later ameliorating. For 4-5 years his memory and mental capacity had deteriorated, and for the last two years he had been impotent. More recently he had experienced urgency of micturition and sometimes incontinence of urine. He was a pale thin man with soft waxy skin and scanty hair of female distribution. Cerebration was slow and somewhat inaccurate. There was bilateral optic atrophy and a bitemporal hemianopia. Apart from these eye-signs, there was some rather indefinite weakness of arms and legs, and spasticity of the left leg.

E.E.G. (Fig. 3).-Alpha rhythm: 12/sec., at about 25 micro volts, but very little could be distinguished.

The main component of the record was a bilateral 5-6/sec. rhythm, almost continuous, of fair regularity, at up to 90 micro volts.

It was not inhibited by eye opening, and was maximal in the pre-central regions.

Operation.-A right trans-ventricular approach revealed a rather large but otherwise normal lateral ventricle, with a patent foramen of Munro. A suprasellar cyst of moderate size was bulging into its floor, but chiefly passing back under the anterior end of the 3rd ventricle. This was drained and its wall removed as far as possible. It appeared to have been roughly spherical and symmetrically placed about the mid-line.
Case 3.-J. F., female, age 22, had a history of 6 years secondary amenorrhoa, 6 months frontal headaches, and 5 months blurring of vision of the left eye and occasional diplopia. Appetite had recently increased and she had gained a stone in weight in a month. She had also been drinking more lately, with attendant polyurea.

When examined she had V.A.R. 6/18. V.A.L. 6/12: and bitemporal visual field defects, chiefly of the upper quadrants. The tone of the left leg was rather greater than that of the right and on the left side she had an equivocal plantar response. The c.s.f. was under a pressure of $200 \mathrm{~mm}$. of c.s.f. and contained $70 \mathrm{mgm}$./ 100 c.c. of protein.

E.E.G. (Fig. 4 (1)).-Alpha Rhythm: 16/sec., regular, at about 20 micro volts. In all positions there was an almost continuous, rather irregular 5-7/sec. rhythm, mostly of fairly low voltage, but up to 50 micro volts. Hyperventilation produced little change.

Operation.-A suprasellar tumour was found, partly cystic, but largely solid. As much as possible was removed and the patient ultimately discharged to another hospital for deep X-ray therapy.

She returned $2 \frac{1}{2}$ months later because of an exacerbation of her headaches. The chief changes noted at this time were: V.A.R. 6/60. V.A.L. 6/18, hypertonus of left arm and leg, with some weakness of dorsiflexion of the left foot and a plantar response on the left side which was definitely extensor.

E.E.G. (Fig. 4 (2) and (3)) showed a bilateral 5/sec. rhythm, at up to 100 micro volts, maximal in the precentral regions, where phase reversal was present on the right side. In the right frontal area there was a gross irregular discharge from $1 / \mathrm{sec}$. to $3 / \mathrm{sec}$, , at up to 150 micro volts, which showed phase-reversal over the frontal pole.

The patient died three weeks after readmission, and the post mortem revealed a tri-lobed cystic tumour, one lobe of which occupied the enlarged pituitary fossa, while one projected forwards and somewhat to the left;

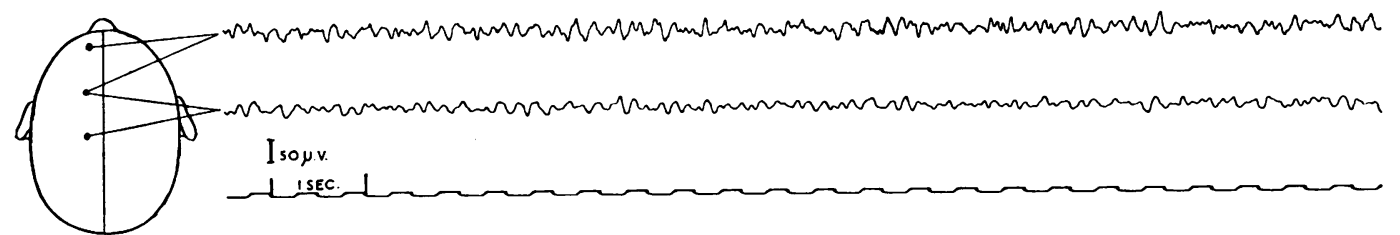

Fig. 3.-Case 2. Showing frontal 5-6/sec. rhythm.

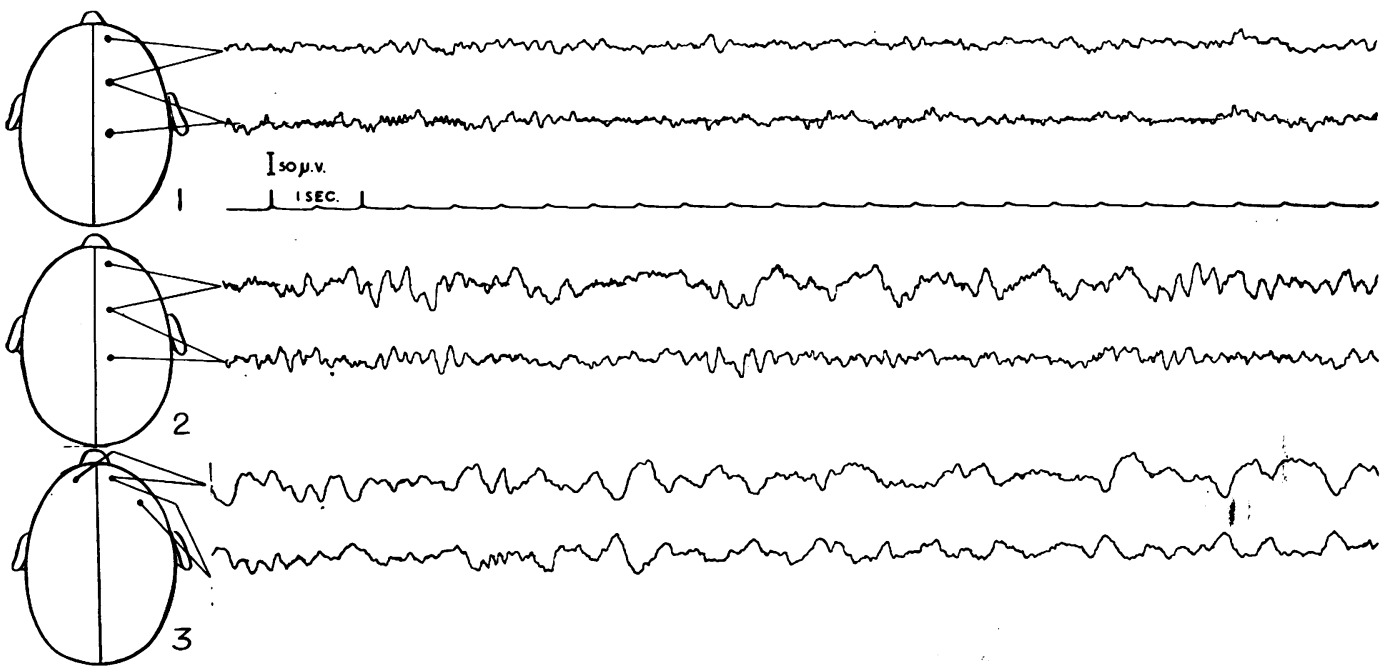

Fig. 4.-Case 3. (1) Showing 16/sec. Alpha-rhythm and 5-7/sec. frontal Theta rhythm. (2) The same position 4 months later showing increased amplitude of $5 / \mathrm{sec}$. rhythm, and also frontal Delta activity. (3) Same date as (2): phase reversal of Delta rhythm over Right frontal pole. 


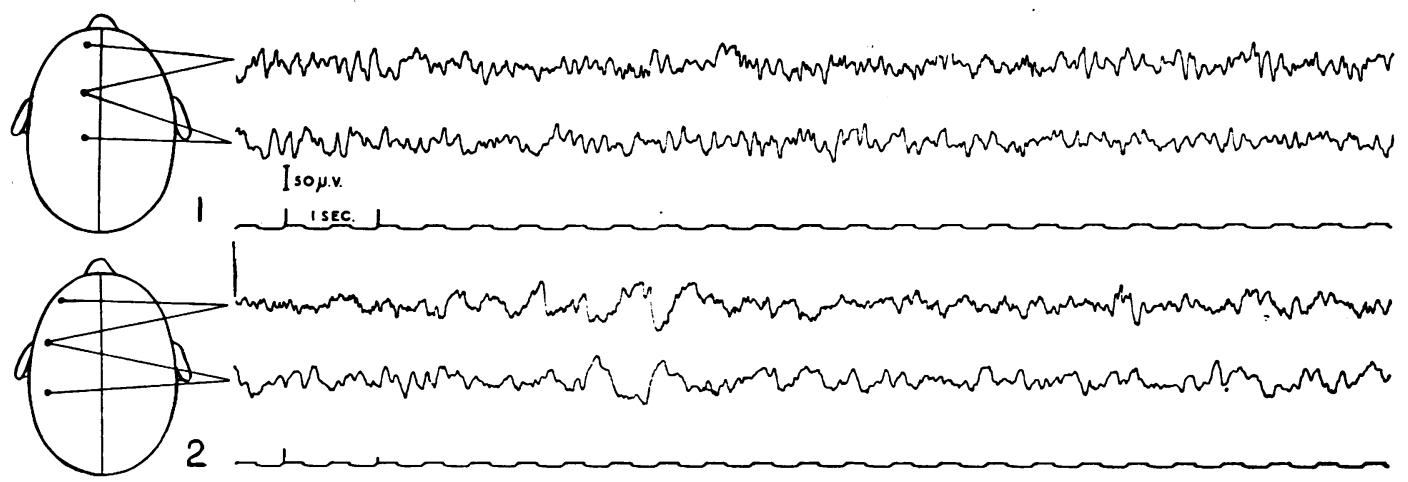

Fig. 5.-Case 4. (1) 6-7/sec. rhythm in left frontal region. (2) Phase-reversal of Delta rhythm in left fronto-temporal region.

the largest lobe, $5 \mathrm{~cm}$. in diameter, projected forwards, upwards and to the right into the right frontal lobe.

Case No. 3 is of particular interest as it illustrates a parallelism between changes in the clinical state, anatomical findings, and the e.e.g. It is also a good example of the asymmetrical Theta rhythm, in this case associated with focal delta activity, and suggests the wider application to which the concept of the Theta rhythm can be placed, as exemplified by the following:

Case 4.-L. C., male, age 39, had a 10-year history of petit mal attacks, which, after six years, began to be interspersed with generalized convulsions, without aura or localizing features, at the rate of 1-2 a week. Three months before admission he began to have attacks of occipital headache, with dizziness, usually occurring on looking up from his work and relieved by returning his head to its original position. In time the headache became a more constant dull frontal ache, and was associated with nausea and vomiting. The chief physical signs were a fairly severe mixed aphasia, mild papilloedema and a right inferior homonymous field defect. There was a mild right hemiparesis, most marked in the face and arm.

E.E.G. (Fig. 5) showed an almost continuous 6-7/sec. rhythm at about 50 micro volts, with phase-reversal in both pre-central para-sagittal positions. There was also an irregular slower discharge of rather higher voltage, maximal on the left side, where phase-reversal occurred in the fronto-temporal region. This was reported as being probably due to a left fronto-temporal basal lesion.

On the evidence of a ventriculogram the tumour was thought to be in the left temporal region, and accordingly this area was explored, rather far back. An area of what appeared to be gliomatous cortex was exposed, but on incising this a firm globular tumour was revealed. This proved to be a meningioma, weighing 46 grams, arising from the dura of the petro-sphenoid ridge, towards its medial end. The tumour was removed, apparently intact, but unfortunately the patient died some months later from an extremely rapidly growing recurrence.

Several cases similar to the above have been seen, and there is every reason to think that the Theta rhythm concept is a useful addition to the accepted methods of tumour localization, but the exact origin and physiological significance of the rhythm are still in doubt. Although the causal lesion is almost certainly basal this does not necessarily imply that one is recording basal potential changes; it is possible that a basal pace-maker is activated by the lesion, and that the potential changes recorded are of cortical origin, though under the influence of this pace-maker. Jasper (1941) has claimed to have recorded basal potentials by means of a sphenoidal electrode, but as this was the middle electrode of three, the other two being symmetrically placed over the frontal cortex, there is no reason why the recorded potential changes should not have occurred at the cortex, provided a common pace-maker is postulated. It is at least probable that the whole e.e.g. is of cortical origin, though under the influence of deeper structures.

The Theta rhythm occurs, of course, in conditions other than basal tumours and is particularly well seen as a component of the very mixed records associated with hydrocephalus of the 3 rd ventricle, and tumours in the ventricle, as exemplified by Case 1. In such records the distribution of the rhythm is usually more posterior than in those of the hypothalamic region, or it may be generalized. It is also fairly frequently seen following concussive head injuries, and Silverman (1943) and Hill (1944) have described bilaterally synchronous $4-7 / \mathrm{sec}$. rhythms associated with aggressive psychopathy, though they respectively locate these as pre-frontal and post-central. These observations are not incompatible with lesions of the hypothalamus (Hill, 1944), and the evidence from the tumour cases points to the hypothalamus and thalamus as the source of the rhythm.

In a few instances a well-defined unilateral 4-7/sec. rhythm has been seen in association with a superficial cerebral tumour. I have regarded this as a "tumour" phenomenon, rather faster than the usual range, but Walter (personal communication) has pointed out that it could be explained by the cutting off of the cortex from thalamic control by the deeper parts of the cumour. This may well be so, but I think it would be a mistake at present to give to certain frequencies values which are too absolute-the most familiar normal rhythm is $10 / \mathrm{sec} . \pm$ about 20 per cent., but $10 / \mathrm{sec}$. rhythms are not necessarily all normal; nor does the alpha rhythm, slowed by trauma to perhaps $7 / \mathrm{sec}$., necessarily differ in character from the normal except in its frequency.

Many tumour records contain waves lying in the 
4-7/sec. range, but not as a clearly defined rhythm; it is possible that they are of the same origin, but, for the utilitarian purpose of localization, a fairly definite rhythm is necessary before one is justified in giving to it the special Theta significance.

\section{Conclusion}

The usefulness of the e.e.g. in tumour localization is beyond doubt, and it has an occasional value as a pointer in tumour differentiation. It is not proposed to compare the respective merits of the various methods of localization because all methods available and suitable should be applied, and regarded as complimentary, but it should be noted that the e.e.g. has one very great advantage over airreplacement methods: it is entirely free of discomfort or danger to the patient. Positive findings in the e.e.g. are probably about as reliable as in either ventriculography or clinical methods, but, as in them, anomalous localizations do occur, sometimes explicable after the event and sometimes not.

The tumours which lend themselves most readily to localization are those of the free convexity which are rapidly growing, but even among these there are some which show only a little diffuse abnormality. From the present series it is possible to choose several pairs with very similar lesions, one of which was easily localized by gross slow activity while the other showed a minimal general change. It may well be that investigation of such negative results will prove as profitable in the elucidation of cerebral behaviour as that of the more spectacular dysrhythmias.

\section{Summary}

Results obtained by e.e.g. in 120 intracranial tumours and 5 abscesses are tabulated and discussed. About 50 per cent. of accessible lesions were correctly localized. It is not considered that cerebellar tumours are normally localizable.

There is a somewhat greater tendency to slowing of the alpha rhythm than in other cases, including acute head injuries. Asymmetry of alpha rhythm is an inconstant but sometimes useful finding.

The record associated with raised intracranial pressure is exceedingly variable, and bears no direct relationship to the height of the pressure nor to the degree of papillœdema, but some to the state of consciousness.

The "ideal " method of localization is the finding of phase-reversal of slow waves, but not infrequently one must depend upon their maximum intensity. Where a number of slow rhythms are present the slowest is usually of major significance for localization.

From the accessible tumours nine normal records were obtained, of which seven were from parasagittal lesions, emphasizing the well-known diffculty in their localization.

Hyperventilation is unlikely to do more than accentuate the features of the resting record.

It is suggested that the $4-7 /$ sec. frequency band or Theta rhythm has a special significance, and is associated with lesions in the region of the third ventricle. A number of cases are described in which this concept has proved of localizing value.

My thanks are due to Mr. Harvey Jackson, whose cases provide the material for this paper, and to E. B. C. Hughes and W. Grey Walter for their advice and criticism. Three of the cases cited were referred to this Hospital by Dr. J. St. C. Elkington, Nos. 1 and 4 from the National Hospital, Queen Square, and No. 3 from St. Thomas's Hospital.

\section{REFERENCES}

Foerster, O., and Altenburger, H. (1935). Deutsch $Z$. Nervenheilk. 135, 277.

Gibbs, F. A., and Gibbs, E. L. (1941). Atlas of Electroencephalography. Boston.

Hill, J. D. N. (1944). Proc. roy. Soc. Med., 37, 317.

Jasper, H. H. (1941). See Penfield and Erickson, Epilepsy \& Cerebral Localization. London.

Silverman, D. (1943). Arch. Neurol. Psychiat (Chicago), $50,18$.

Smith, J. R., Walter, C. W. P., and Laidlaw, R. W. (1940). Ibid., 43, 472.

Walter, W. G. (1936). Lancet (ii), 305.

Will (1937). Proc. roy. Soc. Med., 30, 579.

Williams, D. (1939). Brain, 62, 321. 